\title{
Charles Sorel, La Bibliothèque française (1667)
}

\section{Laura Rescia}

\section{(2) OpenEdition}

\section{Journals}

\section{Edizione digitale}

URL: http://journals.openedition.org/studifrancesi/5321

DOI: 10.4000/studifrancesi.5321

ISSN: 2421-5856

\section{Editore}

Rosenberg \& Sellier

\section{Edizione cartacea}

Data di pubblicazione: 1 dicembre 2016

Paginazione: 525-526

ISSN: 0039-2944

\section{Notizia bibliografica digitale}

Laura Rescia, « Charles Sorel, La Bibliothèque française (1667)», Studi Francesi [Online], 180 (LX | III) |

2016, online dal 01 janvier 2017, consultato il 18 septembre 2020. URL : http://

journals.openedition.org/studifrancesi/5321 ; DOI : https://doi.org/10.4000/studifrancesi.5321

\section{Questo documento è stato generato automaticamente il 18 settembre 2020.}

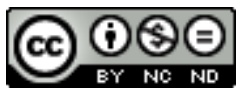

Studi Francesi è distribuita con Licenza Creative Commons Attribuzione - Non commerciale - Non opere derivate 4.0 Internazionale. 


\title{
Charles Sorel, La Bibliothèque française (1667)
}

\author{
Laura Rescia
}

\section{NOTIZIA}

CHARLES SOREL, La Bibliothèque française (1667), édition critique réalisée par F. D’angelo, M. Bombart, L. Giavarini, C. Nédélec, D. Ribard, M. Rosellini, A. Viala, Paris, Honoré Champion éditeur, 2015, $680 \mathrm{pp}$.

1 Ben nota ai dix-septiémistes, che la utilizzano sovente come catalogo della produzione libraria del XVII secolo, o per trarne definizioni e chiarimenti relativi alla percezione di un genere letterario specifico, quest'opera soreliana, benché da tempo riabilitata come l'intero corpus dell'opera di Charles Sorel, non aveva finora suscitato interesse critico fatta eccezione per un articolo apparso nel 1979. Questa edizione critica moderna, allestita da un gruppo di soreliani, che si inserisce nelle nuove edizioni dell'opera di Sorel intraprese dall'editore Champion, ha, tra i suoi molti meriti, quello di restituirci un libro che merita attenzione in quanto progetto a sé stante, e non solamente come repertorio bibliografico. È quanto sottolineano gli autori dell'Introduction (A. Viala e M. Rosellini), che inquadrano in primo luogo il testo in relazione alla sua dimensione storica. La Bibliothèque française appare negli anni Sessanta del secolo, allorquando si assiste da un lato alla moltiplicazione delle querelles letterarie, dall'altro a un curioso fenomeno, ovvero alla percezione di un aumento dell'offerta libraria, proprio quando l'editoria conosce una sua prima e significativa crisi. Spiegabile come riflesso di quanto accadeva all'inizio del secolo, quando la prima volta nella storia del libro si rendevano disponibili un gran numero di testi di natura diversa, e in particolare di testi narrativi, la sensazione di una inarrestabile valanga di novità è probabilmente tra le cause scatenanti il progetto soreliano, che intende offrire ai suoi lettori, come si legge nel sottotitolo «l'Examen et le Choix des meilleurs et des principaux livres de notre Langue» In questa «bibliothèque sans murs», come l'autore stesso la denomina nel suo Avant-discours, Sorel agisce recuperando in parte una tradizione già consolidata, ma 
soprattutto innovando questo lascito nella direzione della modernità. Se infatti il genere in cui si cimenta non è nuovo - Sorel cita Conrad Gesner e la sua Bibliotheca universalis (1545) come predecessore eminente a cui riferirsi - vero è che, a differenza di Gesner, il nostro autore intende perseguire il progetto di dimostrazione dell'abilità della lingua francese-e non più degli idiomi classici-per veicolare il sapere, spostandosi dunque nel campo dei promulgatori della modernità. E se i suoi predecessori compatrioti, La Croix du Maine e Du Verdier avevano già presentato le loro Bibliothèques françaises rispettivamente nel 1584 e 1585, Sorel se ne distanzia, reclamando la sua originalità sotto tre diversi profili. Invece di inseguire un'illusoria esaustività, Sorel infatti opta per la selezione dei titoli, che vengono presentati non già in ordine alfabetico, bensì per materia (grammatica, eloquenza, filosofia...) e, tratto innovativo, per genere letterario - forse, suggeriscono i prefatori, aderendo al modello già sperimentato da Gabriel Naudé per il suo Avis pour dresser une bibliothèque del 1627. La propensione alla modernità è il tratto più evidente di questo testo, che privilegia $\mathrm{i}$ libri pubblicati a partire dalla seconda metà del XVI secolo. Il progetto soreliano, che già appariva tra le righe del Francion e del Berger Extravagant, due opere nelle quali l'autore si rivela straordinariamente consapevole della dimensione sociologica della vita letteraria, è originale anche sotto un altro profilo. Per rispondere al criterio di utilità, che tanta centralità assume in tutto il suo pensiero, questa Bibliothèque non si rivolge all'honnête homme, ma all'homme ordinaire abbandonando l'elitarismo del pensiero libertino per avvicinarsi a quell'universalismo che caratterizzerà il discorso del Secolo dei Lumi. Proprio per questo pubblico, per renderlo maggiormente consapevole dello sviluppo della storia dei generi affrontati, Sorel fa così evolvere il suo repertorio da semplice bibliografia a vera e propria storia letteraria.

2 I criteri di edizione del testo privilegiano l'edizione del 1667, più estesa nel paratesto, pur offrendo in nota le varianti - peraltro non sostanziali - dell'edizione del 1664. Per quanto riguarda lo spinoso problema della modernizzazione, si è optato per offrire un testo maggiormente leggibile, ma tuttavia rispettoso da un lato delle particolarità lessicali, quando queste rivelano delle sfumature semantiche che la modernizzazione dei lemmi avrebbe cancellato, dall'altro per la conservazione sia della punteggiatura, sia, in linea di massima, delle scelte tipografiche. Le annotazioni critiche si rivelano precise e puntuali nell'illuminare passaggi o singoli lemmi oscuri, riferimenti storici e rinvii bibliografici-eccezion fatta per le opere facilmente reperibili a partire dal catalogo della Bibliothèque nationale de France.

Il testo soreliano è seguito da un ricco dossier, composto da una serie di articoli curati dai singoli editori che hanno partecipato a quest'opera collettiva, ognuno apportando le proprie specifiche competenze, che poi si esplicitano nelle diverse direzioni di lettura del testo. Dinah RIBARD, Un manuel de combat, pp. 465-485, esamina la dispositio che, messa in relazione alle numerose riflessioni contenute in apertura o in chiusura dei diversi capitoli, le permette di precisare la posizione soreliana per quanto riguarda $\mathrm{i}$ criteri di valutazione delle discipline e dei saperi ("sciences" e "arts"). Non al pubblico mondano o di corte è delegata tale stima, che nell'intentio auctoris dovrebbe invece poggiare sulla qualità stessa dei libri presi in esame. Mathilde BOMBART, Un regard sur le monde littéraire: les "Gens de Lettres» et leurs querelles, pp.487-506, si concentra sullo spazio dedicato alle querelles, nonché al problema dell'autorialità; ne emerge una posizione originale del nostro autore che, nel primo caso, sembra valorizzare assai più la reazione del pubblico che quella dei savants, e nel secondo assume la possibilità di 
rivendicare la paternità dei suoi scritti come una delle strategie possibili, anche se non la sola, sottolineando dunque implicitamente l'autonomia delle proprie scelte. Claudine NÉDELEC, Style, langue, énonciation pp. 507-529, effettua un'analisi delle posizioni soreliane rispetto alle due nozioni di stile e lingua, e alla sua pratica enunciativa, per sottolinearne l'originalità rispetto alla cultura dominante. Filippo D'ANGELO, Du bon usage des fictions pp. 531-544, torna sull'annoso problema dell'opinione soreliana rispetto alla finzione narrativa, osservandone l'evoluzione attraverso il concetto di vraisemblance applicato alle histoires comiques, e giungendo a ipotizzare una rivalorizzazione della finzione, messa in relazione alla storia, che Sorel avrebbe intrapreso per far rivalutare l'insieme della sua opera. Michèle ROSELLINI, «La Guide de l'Histoire de France»: une contribution à l'éducation politique des lecteurs? pp. 545-580, si concentra sulla concezione della storia nei suoi testi teorici, ponendola in connessione alla sua vicenda di storiografo reale, al concetto di générosité, che assume valenza politica, alle qualità dello storico e del pubblico, altrettanti elementi che depongono a favore della modernità della sua concezione. Laurence GIAVARINI, L'ordre des noms et l'auctorialité de Sorel selon "La Bibliothèque française" pp.581-607, ritorna sulla struttura del testo, che non adotta un ordine alfabetico, e sulla pratica innovativa di rompere il binomio autore-titolo, prassi che l'A. mette in relazione con la riflessione foucaultiana sulla funzione autoriale, che Sorel realizza grazie al gioco enunciativo irrispettoso della norma sociale, e interpretabile come "désir d'histoire sur une sorte d'atopie institutionnelle qui le rend fragile». Chiudono il volume una bibliografia dell'opera soreliana, a cura di L. GIAVARINI, redatta a partire dalle riflessioni dell'autore contenute nella Bibliothèque, una succinta bibliografia critica e un indice dei nomi d'autore, letterati e filosofi. 